\title{
Bienes patrimoniales en el turismo cultural Un acercamiento a su estado de investigación
}

Recibido: 21/02/17 - Aceptado: 11/04/17

\author{
José Antonio Uribe Esquivel* \\ Maribel Osorio García \\ Universidad Autónoma del Estado de México
}

\section{Resumen}

El patrimonio, en sus diversas expresiones, se ha desarrollado -junto con el turismo cultural- como una de las opciones más importantes del accionar turístico, razón por la cual se han efectuado variadas investigaciones sobre la relación entre ambos; sin embargo, los bienes patrimoniales han cobrado gran relevancia para su estudio particular. A fin de realizar una caracterización sobre su estado de investigación, se ha revisado una serie de documentos recopilados de tres revistas con alta presencia de publicaciones en torno al tema, que dio como resultado la delimitación de cuatro líneas temáticas: bienes patrimoniales e identidad social, aprovechamiento y viabilidad de los bienes patrimoniales como recursos turísticos, imaginarios turísticos sobre los bienes patrimoniales, y recuperación y restauración de los bienes patrimoniales para su aprovechamiento turístico. Otro aspecto identificado es la predominancia del enfoque metodológico cualitativo con el cual se realizan las investigaciones analizadas, así como la importancia de países como Malasia, Turquía y España en la aportación de trabajos de investigación.

Palabras clave: Bienes patrimoniales, turismo cultural, estado de investigación.

*Correos electrónicos: antonio.uribesq93@gmail.com, maribelosorio2@gmail.com 


\title{
Heritage assets in cultural tourism An approach to their state of research
}

Recieved: 21/02/17 - Accepted: 11/04/17

\author{
José Antonio Uribe Esquivel* \\ Maribel Osorio García \\ Universidad Autónoma del Estado de México
}

\begin{abstract}
Heritage in its various expressions has been developed together with cultural tourism as one of the most important options in tourism activities, reason why various research studies have been conducted about the relationship between them, nevertheless heritage assets have reached relevance for their specific study. In order to establish a characterization about its research status, a set of documents gathered from three magazines with high content on the topic, allowed the delimitation of four thematic lines: heritage assets and social identity, use and viability of heritage assets as touristic resources, touristic imaginaries related to heritage assets; and, recovery and restoration of the heritage assets for their touristic exploitation. Another identified aspect is the predominance of the qualitative methodological approach under which research is conducted in the field, as well as the importance of countries like Malaysia, Turkey and Spain in terms of the contribution of research documents.
\end{abstract}

Key words: Heritage assets, cultural tourism, state of research.

*E-mails: antonio.uribesq93@gmail.com, maribelosorio2@gmail.com 


\section{Introducción}

Los bienes patrimoniales y el turismo cultural podrían considerarse como una misma entidad (Fernández Poncela, 2011), pero recientemente se han diferenciado en la escena turística de una manera consciente y reflexiva por parte de las comunidades en las que se encuentran tales bienes. Esta nueva propuesta de valoración y análisis de las necesidades de los propios bienes patrimoniales se ha transformado en una tendencia creciente en el turismo y, por lo tanto, en las investigaciones realizadas para esta disciplina.

La estrategia de conservación establecida por la Organización de las Naciones Unidas para la Educación, la Ciencia y la Cultura (Unesco) para declarar como patrimonio mundial de la humanidad los bienes y sitios naturales y culturales en peligro (Unesco, 2013) dio origen a una revaloración de todos aquellos recursos que mostraran un valor histórico, cultural o natural singular, fomentando en el nivel internacional la conservación y protección de los bienes patrimoniales, en especial de los que forman parte de la selecta lista. Su articulación con el turismo ha ido en aumento, puesto que se han convertido en uno de los mayores atractivos de los flujos turísticos, tanto de la modalidad cultural como de la natural. Su relevancia es tal que el número de sitios patrimonio de la humanidad de la Unesco (2009) es un indicador considerado para obtener el posicionamiento en el índice de competitividad turística por país, editado por el World Economic Forum (2015) cada año, y que ubica a Italia, España, Francia y Alemania como los países que en 2015 tenían el mayor número de sitios con esta categoría.

En particular para los bienes culturales se ha denotado una acentuada relación entre los bienes patrimoniales y la identidad comunitaria. Todos aquellos elementos patrimoniales culturales tienen, han tenido y tendrán un gran impacto en distintos niveles de las estructuras sociales otorgándoles una identidad comunal o colectiva, lo cual es incluso más importante que el bien mismo, ya que este último depende del valor que le confiera la sociedad en la que se desarrolla y sus formas de aprovechamiento, entre ellas, como recurso turístico.

En consecuencia, se ha dado una importancia cada vez mayor a la relación bienes patrimoniales-comunidad-turismo, en la cual se postula que el turismo debe ser complemento de otras actividades enfocadas a generar o reforzar la 
identidad de una comunidad mediante la interpretación de sus bienes patrimoniales culturales, para después potencializarlos en el turismo. De aquí el interés por estudiar específicamente los bienes patrimoniales mediante una aproximación a la literatura científica enfocada a ellos y su conexión con el turismo cultural. El objetivo de este trabajo es caracterizar el estado de la investigación de ese tema a través de sus líneas temáticas, fundamentos teóricos, herramientas metodológicas y sus principales centros de producción con el propósito de distinguir sus tendencias y principales retos.

La presente investigación se ha estructurado en cuatro apartados: el primero ofrece un marco conceptual en el que se incluyen los bienes patrimoniales y el turismo cultural como principales categorías; el segundo consiste en un marco metodológico en el que se expone la situación cuantitativa de la producción científica del tema en cuestión y la evolución temporal que este ha experimentado, para más adelante explicar el enfoque y la técnica metodológica con los que se aborda la investigación; en el tercero se da cuenta de la caracterización de las investigaciones identificadas y analizadas, las cuales se clasificaron en cuatro líneas temáticas; y se concluye con un apartado de consideraciones finales sobre el estado de investigación de los bienes patrimoniales.

\section{Marco conceptual}

Al revisar los términos "bienes patrimoniales" y "turismo cultural", se observa que existen elementos afines en sus respectivos conceptos, lo que permite un mejor entendimiento sobre ellos. No está de más mencionar que la tarea de conceptualizar y analizar esos términos requiere un trabajo de investigación propio, y de las mismas proporciones que el presente, por lo que solo se hace referencia a aquellos elementos de trascendencia y afinidad con esta investigación.

Los elementos clave para entender el concepto bienes patrimoniales comienzan por resaltar la procedencia de tales bienes, la cual es atribuida a un ente creador (artista, músico, arquitecto, etc.) que no solo le otorga una existencia (material o no), sino que en ella refleja a su vez un alma colectiva (social) y la dota de una serie de valores populares que le confiere cierta vitalidad a esa creación, la cual permite expresar las circunstancias y realidades sociales que permean el entorno de una comunidad (Unesco, 1982; омт cit. en Universidad Interamericana para el Desarrollo, s. f.; Laws of Malaysia, 2006; y Orbasli, 2000). 
Otro elemento que sobresale del análisis del término en cuestión es el enfoque con el que se aborda en el campo de la semántica y la lingüística de diversos idiomas; desde estas perspectivas, lo que abarcan los bienes patrimoniales (patrimonio) se ramifica, dejando atrás el entendimiento clásico que lo define como una creación o una extensión de la conciencia social; ahora se le otorga un entendimiento físico o incorpóreo (se menciona incorpóreo en lugar de inmaterial debido a que pese a no poseer un límite físico o material no confina su existencia espiritual); tal enfoque le confiere asimismo un valor de pertenencia del ser, ya que puede distinguirse como una reliquia (algo precioso que debe ser cuidado), una riqueza (espiritual) o una propiedad; y finalmente lo dota de una temporalidad causal, ya que se considera como un legado que "ha pasado", como un esfuerzo para representar el presente mediante su comprensión histórica, o como el resultado de factores y situaciones históricas, de acuerdo con García Jiménez (2010), Davison cit. en Mohd, Ahmad y Syed (2013), y Tunbridge y Ashworth (1996).

Para el caso de turismo cultural, se han aislado elementos de diversas concepciones del término. El principal hace énfasis en el aprovechamiento de los bienes patrimoniales y su utilización como atracciones turísticas, según afirman la World Tourism Organization (2008), Tümertekin, Emekli y Dogăner cit. en Akbulut y Artvinli (2011), Smith (1979), y André, Cortés y López (2003). Otro elemento que se repite en varias definiciones es el que califica al turismo cultural como un medio o canal de aprendizaje del patrimonio y sus comunidades, como lo expresan McIntosh, Goeldner y Ritchie (1995) y el documento Creative Nation: Commonwealth Cultural Policy (1994), emitido por el Departamento de Comunicaciones y Artes de Australia.

Mediante estas conceptualizaciones se ha esbozado la relevancia de los bienes patrimoniales en el desarrollo de la actividad turística, esencialmente en la modalidad de turismo cultural y, lo más importante, se ha vislumbrado que, al reconocer estos bienes, las sociedades podrán potencializarlos en la escena turística. De la misma manera, y como primer acercamiento, se comprende a los bienes patrimoniales no solo como entidades físicas u objetos que persisten y trascienden el paso del tiempo, sino también como elementos propios de las condiciones humanas que los generan, y como generadores de realidades sociales. 


\section{Marco metodológico}

Para determinar los textos científicos a revisar, se llevó a cabo una primera exploración en la base de datos Science Direct, considerada la opción más viable por el tipo, cantidad y accesibilidad del contenido para la investigación; se aplicó un filtro temporal del año 2000 al 2014, y se encontró un total de 36164 artículos sobre el tema de turismo en general, de los cuales 4884 tratan sobre los bienes patrimoniales y el turismo cultural, lo que representó $12 \%$ de los trabajos producidos.

Cabe destacar que la producción de textos científicos en torno al tema de turismo cultural ha mostrado un crecimiento durante el periodo de estudio (figura 1).

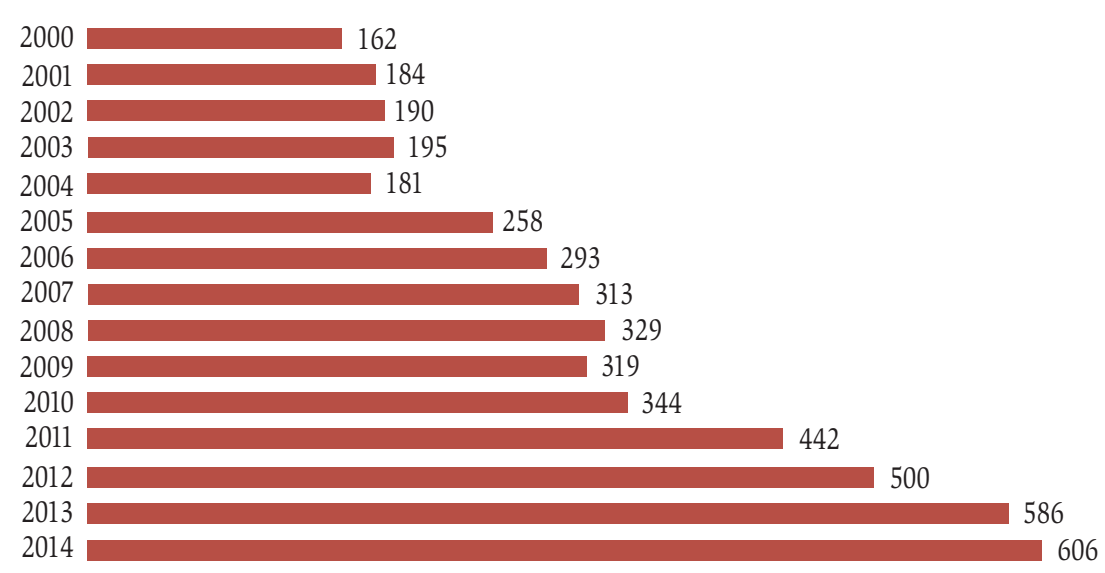

Fuente: Elaboración Propia con base en la información de Science Direct (consulta realizada el 08/09/16).

Figura 1. Textos científicos publicados en Science Direct 2000-2014

Dentro de las revistas indizadas en la base de datos que contienen artículos relacionados con el tema de estudio, las que contienen el mayor número de artículos son tanto del campo del turismo: Tourism Management y Annals of Tourism Research, como de otros campos: Procedia - Social and Behavioral Sciences, Land Use Policy, Ecological Economics, Cities y Journal of Cultural Heritage (figura 2). 


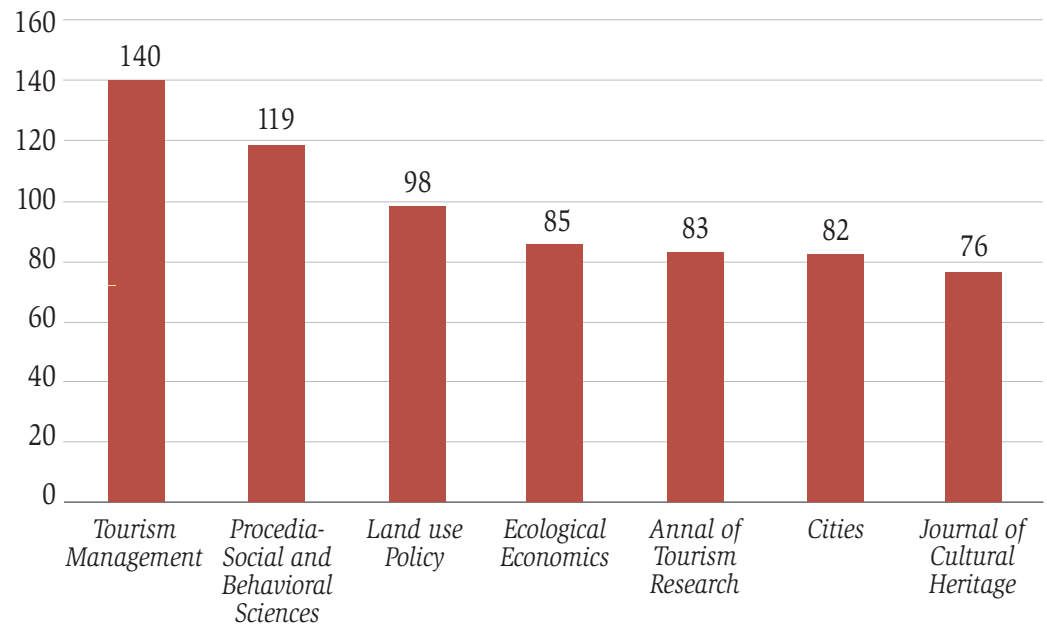

Fuente: Elaboración propia con base en la información de Science Direct (consulta realizada el 08/09/16).

Figura 2. Textos científicos publicados por revista indizada Base Science Direct

Para contar con un panorama de la producción científica iberoamericana vinculada con el tema, se consultó la base de datos Red de Revistas Científicas de América Latina y el Caribe, España y Portugal (Redalyc), considerada por la afinidad cultural de las investigaciones de la región iberoamericana; se acotó el espectro de búsqueda de los años 2000 a 2014; se encontraron 1440 artículos relacionados con el turismo cultural y, de ellos, 383 con los bienes patrimoniales, los cuales representan $21 \%$.

Lo anterior demuestra que la producción de artículos relacionados con el estudio de los bienes patrimoniales y el turismo cultural en Iberoamérica posee una mayor consideración para los investigadores de la materia turística que la producción en el plano mundial.

En el caso del número de publicaciones realizadas por año sobre el tema, en la base de datos Redalyc se observa un crecimiento notable pero un tanto errático marcado por años específicos en los cuales las investigaciones fueron efectuadas con mayor frecuencia como 2008, 2010, 2011 y 2013 (figura 3). 


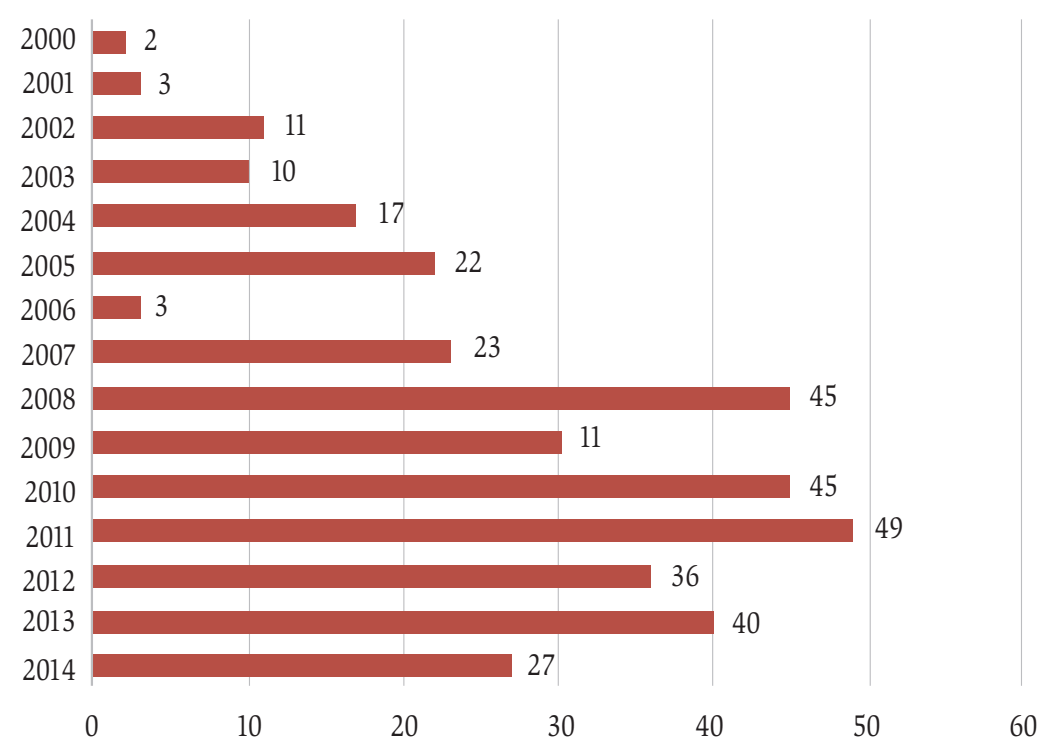

Fuente: Elaboración propia con base en la información de RedAlyc (consulta realizada el 11/09/16).

FiguRa 3. Textos científicos publicados en RedAlyc, 2000-2014

En contraposición con lo obtenido de las publicaciones incluidas en Science Direct, aquellas de Redalyc con mayor aportación están ampliamente relacionadas con la materia turística, como PASOS. Revista de Turismo y Patrimonio Cultural y Cuadernos de Turismo; pero de igual manera se identifican revistas que no pertenecen al campo del turismo y se centran en otras áreas del conocimiento, como Chungara, Revista de Antropología Chilena, que, como su nombre lo indica, se enfoca a estudios antropológicos (figura 4).

Como pudo observarse, el estudio del tema en cuestión ha adquirido mayor importancia no solo para el campo de conocimiento del turismo, sino para otras áreas, de acuerdo con las revistas indizadas mencionadas.

Una vez realizada esta indagación, se eligieron las revistas con mayor número de artículos sobre los bienes patrimoniales para elaborar el presente estado de investigación. Respecto a Science Direct, se escogió Tourism Management, sin embargo, los artículos no se encontraron en la opción de acceso abierto, por 
lo que se optó por trabajar con Procedia - Social and Behavioral Sciences, la segunda de mayor número de artículos. En cuanto a Redalyc, se eligió PASOS. Revista de Turismo y Patrimonio Cultural, asumiéndola como la líder sobre esta temática en Latinoamérica. Con el propósito de tener un acercamiento a la producción mexicana en torno a este tema, se decidió incorporar El Periplo Sustentable en la revisión de artículos.

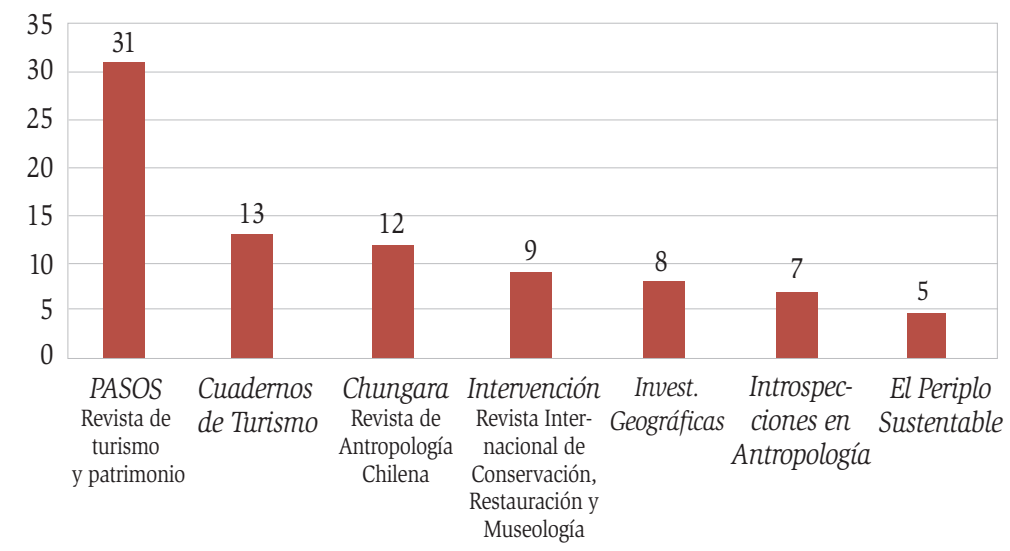

Fuente: Elaboración propia con base en la información de RedAlyc (consulta realizada el 11/09/16).

FigURA 4. Textos científicos publicados por revista indizada. Base RedAlyc

Definidas las revistas de las cuales se extraerían los artículos objeto de estudio, se hizo una lista de los publicados entre 2010 y 2014, y se verificó que tocaran el tema de los bienes patrimoniales. De Procedia - Social and Behavioral Sciences se retomaron 17 artículos procedentes de diversos países, cuyos temas se enfocaban en los recursos culturales y el turismo; en PASOS. Revista de Turismo y Patrimonio Cultural, solo se ubicaron cinco artículos, y de El Periplo Sustentable se eligieron seis. Una vez recabado el material, se aplicó la técnica de análisis de contenido que por sus características permite realizar una revisión objetiva, sistemática, cualitativa y general de las unidades de análisis establecidas para la investigación (Hernández, Fernández y Baptista, 2014; Dwyer, Gill y Seetaram, 2012). 
El procedimiento de análisis de contenido consistió en revisar el material elegido con el propósito de esbozar un panorama general del tema y generar asimilaciones y agrupaciones de términos afines. Posteriormente, se clasificó el material conforme a criterios básicos (criterios flexibles dependiendo de las necesidades de las investigaciones y la literatura), es decir, se llevó a cabo un primer intento por discernir y codificar las líneas temáticas como unidades conceptuales resultantes. Una vez extraídos y aislados los patrones conceptuales afines en los documentos, se estructuraron las líneas sobre las que se generaron las descripciones de las investigaciones revisadas. Finalmente, se dio pauta al discurso explicativo de las líneas temáticas, los resultados y el entendimiento de la cuestión descrita. A continuación se presentan los resultados obtenidos.

\section{Líneas temáticas de los bienes patrimoniales en el turismo cultural}

De acuerdo con las similitudes en las investigaciones revisadas, fue posible conformar cuatro líneas temáticas nombradas (codificadas) de la siguiente manera: bienes patrimoniales e identidad social; aprovechamiento y viabilidad de los bienes patrimoniales como recursos turísticos; imaginarios turísticos sobre los bienes patrimoniales; y recuperación y restauración de los bienes patrimoniales para su aprovechamiento turístico.

\section{a) Bienes patrimoniales e identidad social}

Con relación al vínculo bienes-identidad, el trabajo de Peñalver (2002) señala que al analizar diversos documentos puede comprenderse que la principal característica para la inmersión de un elemento bien patrimonial en el turismo es el reconocimiento y la aceptación colectiva de un elemento cultural (que se encuentre presente en la sociedad que lo valoriza) para su posterior aprovechamiento como elemento de atracción del turismo. El autor enfatiza que dentro de la vasta gama de elementos culturales, la arquitectura es uno de los más comunes, a la cual se le reconoce y adjudica una importancia histórica o social dentro de la evolución de un grupo social o comunidad en un espacio temporal específico.

Otro estudio relevante para identificar los elementos patrimoniales es el de Velasco (2009), en el que analiza el énfasis de la identificación social hacia los 
bienes patrimoniales. Afirma que esta es una característica vital para el aprovechamiento de esos bienes en términos turísticos, ya que una vez identificados como valiosos para la sociedad en la que se encuentran, se propone evaluarlos y considerar su viabilidad turística, con objeto de lograr que en la relación patrimonio-turismo aumente la funcionalidad turística de un bien sin agredir su naturaleza o funcionalidad.

Sobre la importancia que la comunidad local le da al patrimonio, Akbulut y Artvinli (2011) realizaron una investigación en la que se resalta que de esto depende su éxito o fracaso como recurso turístico. En este caso, el objetivo es conseguir que tal comunidad se interese previamente en sus propios elementos patrimoniales, no con un fin turístico, sino más bien identitario, para su posterior reenfoque a los visitantes. Esta investigación afirma que debe despertarse el interés de los pobladores locales antes de considerar siquiera potenciarlo en personas ajenas (turistas).

El aumento de la calidad de vida de los miembros de las sociedades se identifica como un beneficio de la concertación de los bienes patrimoniales en el sistema identitario social; se hace énfasis en las minorías o grupos rezagados por el común social. Serrano, Zarza y Serrano (2013) enfocan su estudio en la participación de las mujeres en museos que sirven como medio de transmisión y conservación de los bienes patrimoniales para la colectividad; en él se describe el aumento de mujeres en tales organizaciones, así como los puestos de mayor importancia que ocupan en ellas.

\section{b) Aprovechamiento y viabilidad de los bienes patrimoniales como recursos turísticos}

Una aportación sobre la activación y, por ende, el aprovechamiento de los bienes patrimoniales la hace García Jiménez (2010); en ella reconoce una paradoja o un nivel de subjetividad, como describen Mallor, González-Gallarza y Fayos (2013) en cuanto a la activación del patrimonio, en diversos estudios y tratados. Los bienes patrimoniales se han dividido, segmentado y clasificado de variadas formas y bajo distintos criterios, pero el principal razonamiento desde el cual se activan es la arbitrariedad, ya que el patrimonio no puede ser visto desde un solo enfoque o perspectiva, debido a que la cultura se desarrolla y evoluciona según necesidades específicas de la sociedad de que se trate, y ello no permite homologar criterios para clasificar los bienes patrimoniales. 
Güngör y Bozyiğit (2011) y Ruiz, Martínez y Verján (2015) identifican que siempre se hace referencia a beneficios o ideales para el turismo como parte del reconocimiento de los bienes patrimoniales, así como también su aprovechamiento para las nuevas modalidades turísticas que evitan la estacionalidad. De igual forma ponderan la modernización de la infraestructura y los servicios turísticos adecuados para cada modalidad turística.

El planteamiento anterior es el mismo de Emekli y Baykal (2011), Kaya y Kurt (2011) y Ataberk y Baykal (2011), quienes refuerzan la premisa de la diversificación de las tipologías turísticas, haciendo énfasis en los bienes patrimoniales, en especial cuando se les ha otorgado un reconocimiento como el de patrimonio cultural de la humanidad por parte de la Unesco, lo que impulsa la actividad turística. Además proponen la hibridación de modalidades turísticas que se adapten a las necesidades del destino objeto de estudio, lo cual representa una nueva posibilidad de aprovechamiento de los recursos naturales y culturales según las necesidades del destino, de los turistas y de la comunidad, para salvaguardar y fomentar el turismo y los beneficios que trae consigo. Así también para los destinos nuevos o no maduros (que presenten la factibilidad) debe buscarse que la oferta de tipologías turísticas sea diversa para aprovechar en mayor medida los recursos existentes, haciendo énfasis en el turismo cultural como la tipología turística en auge.

Marinoski y Korunovski (2012) sugieren el desarrollo de clústeres turísticos en aquellos destinos que ya están inmersos en el turismo, pero que no han obtenido los resultados esperados. La premisa principal de su propuesta es crear clústeres regionales, cada uno diseñado a partir del reconocimiento de los recursos y las necesidades de las subregiones, que a su vez den pauta a un clúster general.

Existen otras circunstancias en las que el turismo cultural es la opción más viable para la reactivación económica, como expone Conesa (2010) en su estudio sobre los destinos mineros de España. En él considera que reconocer e impulsar los bienes patrimoniales que surgieron a la par del desarrollo de la actividad minera en una comunidad, y que fueron asimilados por los miembros de esta, puede aprovecharse mediante el turismo para dinamizar la economía interna y dar a conocer su legado cultural. El autor advierte que los bienes patrimoniales en materia de turismo enfrentan problemas como su desconocimiento 
por parte de los miembros de esas comunidades; el bajo nivel de atractivo por diferencias en los estándares de belleza; el alto costo que supone su restauración o adecuación por su extensión, y su distante localización de centros y circuitos turísticos tradicionales.

Algunos autores identifican las problemáticas que enfrenta el patrimonio en cuanto a su aprovechamiento y su puesta en marcha turística: en su estudio, Prats (2011) describe que luego del análisis de diversos proyectos turísticos se observa que su tendencia hacia el fracaso se debe mayormente a que se olvida la integración total de los componentes de un proyecto complejo, y solo se enfoca en la parte turística, cuestión que considera causa de que el patrimonio sea "profanado" en aquellos proyectos formulados únicamente desde una racionalidad turística sin tener en cuenta sus diversos valores (histórico, social o estético). En opinión del autor, esta tendencia al fracaso es combatida por medio de técnicas y estrategias que pueden parecer "prácticas", pero generan otros problemas, pues no satisfacen las necesidades de los mismos bienes patrimoniales y continúan enfocándose en el aspecto turístico. Esto no quiere decir que el turismo y el patrimonio sean incompatibles: es factible lograr un desarrollo, siempre y cuando los proyectos turísticos consideren los intereses de todos sus elementos (turísticos y patrimoniales). Mohd et al. (2013) señalan como otra problemática del turismo cultural la banalización o dumbing-down de los recursos patrimoniales culturales, debido a la falta de habilidades para transmitir una adecuada interpretación y así se rebaja su significado identitario.

\section{c) Imaginarios turísticos sobre los bienes patrimoniales}

Una aportación destacada es la de Donaire (2012 cit. en Pillet, 2014), quien asegura que el turismo, al aprovechar diversos elementos culturales, puede caer en la idea de la conversión de la cultura en mercancía. Esto es resultado de la propia demanda, la cual no solo busca los elementos ya conocidos, sino también experiencias diferenciadas. Por tanto, el turismo es capaz de crear productos mediante el reconocimiento de distintos elementos como el paisaje, al que se le otorga un valor patrimonial cultural para su aprovechamiento. Como claro ejemplo se considera el paisaje literario, capaz de motivar o generar un desplazamiento: un autor transmite, recrea, crea y transforma un paisaje existente o idealiza uno mediante el uso de la prosa poética, lo que en ocasiones 
hace al paisaje protagonista de su novela. Pillet (2014) hace un recuento de $E l$ Quijote, obra en la cual la descripción paisajística de ciudades hispanas logró crear una ruta turística, compleja y aceptada, integrando los elementos ya existentes o reales a aquellos imaginarios o plasmados en la descripción de Miguel de Cervantes.

En el tema de patrimonio e imaginarios turísticos, Prats (2003) enfatizó que el binomio patrimonio-turismo ha cobrado un papel importante como posicionador en el pensamiento del turista. Por ello se han orientado numerosos esfuerzos a crear proyectos turísticos que involucren los elementos patrimoniales como parte de una oferta, pero se ha generado sobreelaboración de proyectos turísticos, los cuales, en su mayoría, solo obedecen intereses político-administrativos. Esto generalmente provoca monopolización al diseñar los proyectos, con los que se busca activar los bienes patrimoniales en el turismo.

Judd (2003) ha analizado y propuesto una nueva forma de hacer turismo en la que este busque experiencias diferenciadas, ya no solo en los enclaves turísticos, los cuales ofrecen experiencias aisladas, industrializadas y controladas, sino que ahora sean experiencias integrales, fuera de los ambientes "Disney", en las que se compartan y absorban los elementos sociales del enclave turístico; en esta propuesta se tiende a adoptar el modelo europeo, el cual encamina un desarrollo armónico de la ciudad en vez de espacios de segregación. Es decir, se propone iniciar una etapa de posturismo, que consiste en la búsqueda de experiencias fuera de una burbuja enclávica, poniendo como ejemplo el Bronx en la ciudad de Nueva York.

Zain (2014) y Hassan (2014) establecen que el mercadeo o marketing turístico (que puede considerarse como el primer generador de imaginarios turísticos) en los destinos culturales o sobre los recursos patrimoniales naturales y culturales tiene un peso importante para el éxito o fracaso de los discursos, métodos y herramientas utilizados para transmitir la información. Al respecto, Zain (2014) analiza los textos turísticos promocionales (тРт, por sus siglas en inglés) y concluye que el factor lingüístico cultural, es decir, el contexto cultural oral o de la comunicación de cada sociedad, resulta clave cuando los TPT están enfocados a una promoción internacional principalmente. Por ende, resume, es necesario realizar una traducción semántica con base en el contexto lingüístico cultural de los mercados meta y dejar atrás la traducción literal del discurso. Así también, 
Korunovski y Marinoski (2012) y Tovar y Alvarado (2010) exponen que para la correcta mercantilización de los recursos patrimoniales en el turismo es preciso generar estrategias mercadotécnicas sustentables, adecuadas para los segmentos de mercado que se busca alcanzar con la selección de las subtipologías turísticas del turismo cultural.

Como principal recurso de atracción en el turismo cultural, Chui Teo, Mohd Khan y Abd Rahim (2014) proponen utilizar las "experiencias turísticas memorables"; dicho de otro modo, aprovechando sus recursos patrimoniales, posicionar un destino en los imaginarios personales y, mediante el word-of-mouth (voca-oído), impulsarlo a los imaginarios turísticos colectivos.

En su análisis, Zerafinas, Mohd y Faizah (2014) plantean que mediante el turismo patrimonial la gente puede concebir imaginarios turísticos y autentificar sus imaginarios nacionales en destinos que cuentan con un reconocimiento como el de ser patrimonio cultural de la humanidad.

Por una parte, Fernández Poncela (2011) identifica que para generar o modificar un imaginario turístico, no solo se requiere un bien patrimonial tangible, sino también puede recurrirse a aquellos recursos intangibles, de manera más específica, a las conmemoraciones históricas de un país o una región. Es decir, aprovechar el legado histórico con el cual "desde el presente exaltamos el pasado y proyectamos expectativas hacia el futuro” (Fernández Poncela, 2011, p. 142), con el fin de consolidar y cumplir los nuevos requerimientos de los turistas culturales, quienes buscan "un tiempo y un lugar más lentos, auténticos y humanos” (Fernández Poncela, 2011, p. 145).

Por otra parte, Mendoza, Umbral y Arévalo (2011) proponen consolidar o incluso crear imaginarios turísticos enfocados en los bienes patrimoniales culturales mediante la interpretación del patrimonio, realizada por los profesionales del turismo, con base en el conocimiento del recurso y de las necesidades de la audiencia, lo que permitirá seleccionar una técnica apropiada para transmitir las interpretaciones de los recursos culturales.

A fin de reforzar este apartado, Zamudio Vega (2012) da como ejemplo la arquitectura, la cual es valorada de diversas formas; por ejemplo, los expertos en los estudios arquitectónicos poseen elementos de valoración más especializados en la materia, pero para el turismo, en el cual pueden o no participar estos actores, los aspectos por valorar son más simples en su ponderación: altura, 
estilo arquitectónico, edad (permanencia temporal), etc. Estos atributos son promocionados turísticamente en los folletos, boletines y otros instrumentos, por lo que se propone influir en los imaginarios relacionados con el turismo posicionando los elementos arquitectónicos en el pensamiento de los visitantes.

\section{d) Recuperación y restauración de los bienes patrimoniales para su aprovechamiento turístico}

En las comunidades existen diversos recursos patrimoniales que, debido al paso del tiempo, las fuerzas de la naturaleza o las acciones del hombre, se han mantenido o modificado, se encuentran en peligro o incluso han desaparecido. Por esta razón, al evaluar los recursos patrimoniales, algunas veces se identifican algunos dañados o perturbados, pero por el valor identitario que les otorga la comunidad, son susceptibles de aprovechamiento turístico.

La investigación realizada por Idajati (2014) es un ejemplo de lo anterior: mediante la recuperación de un recurso natural, el cual generó una identidad cultural a través de la historia, se plantea rescatar algunos valores culturales afectados por la degradación del bien natural, como el sistema de transporte tradicional, algunas actividades económicas y comportamientos sociales colectivos que se desarrollaban en los alrededores del recurso; tal recuperación se plantea mediante el turismo cultural y la planificación turística.

Es necesario, previo al aprovechamiento de los bienes patrimoniales, realizar una valoración, como proponen López, Arcila, Micossi y Cano (2013) en su evaluación de los templos de Jerez. Los autores postulan que mediante la correcta identificación y evaluación de los recursos puede evitarse su mala gestión, especialmente cuando se encuentran en un estado de alta fragilidad. Una vez evaluada su condición, es posible ponderar su potencialidad turística; para esto plantean identificar los valores presentes en cada atractivo: el histórico, que comprende otros valores como el informativo, el testimonial y el documental; y el artístico, que incluye el arquitectónico, el escultórico y el pictórico.

De la misma forma, Zamil, Hafiz y Abd Rashid (2014) y Martha y Kotzaki (2014) postulan que aquellos elementos cuya singularidad trasciende el valor estilístico que poseen, debido a la influencia y mezcla de elementos culturales de variadas sociedades, son susceptibles de ser recuperados y restaurados para demostrar y proyectar características culturales de una comunidad ancestral. 
Considerando que los recursos naturales propician y moldean, o modifican los sistemas culturales de una sociedad a través del tiempo, Luekveerawattana (2012) añade que esta recuperación de los bienes patrimoniales debe realizarse por un interés social, antropológico y de identidad en primera instancia, en lugar de atender a una razón turística. Mestre (2010), por su parte, advierte que los museos son esenciales para recuperar, restaurar, preservar y estudiar los bienes patrimoniales (tangibles e intangibles), además ayudan a transmitir sentimientos de pertenencia a una comunidad, ya que su principal objetivo no son las exposiciones, sino la recuperación de la identidad y su representación cultural, lo que se refleja en el turismo.

La puesta en escena turística de los bienes patrimoniales, de recursos que han sido restaurados, es susceptible de modificación debido a que estos dependen de un contexto humano, e incluso emocional, de quienes realizan las tareas de restauración; por otro lado, el visitante prefiere que tales bienes permanezcan apegados a la tradición que los generó, es decir, que mantengan su valor histórico, único y de singularidad ancestral. Es entonces cuando estos bienes se ven conflictuados por las necesidades evolutivas (en su interpretación) y los requerimientos turísticos de los visitantes. Rahmawati, Supriharjo, Setiawan y Pradinie (2014) califican esta problemática como resilience, que, aplicada al turismo, se caracteriza como la habilidad para mantener una estructura (social o material), patrones de comportamiento (expresiones culturales) y la interpretación de los mismos para que puedan persistir y adaptarse a las nuevas necesidades sin perder la esencia y, por ende, su atractivo para el turismo.

Los bienes y recursos culturales son capaces de posicionarse por sí mismos en los imaginarios turísticos, como se ha mencionado con anterioridad: las personas idealizan y generan una expectativa sobre los recursos. Por ello, la restauración de los bienes patrimoniales se ha convertido en una de las tareas más importantes para el turismo: al identificar el valor de cada recurso, se le da una perspectiva turística; como consecuencia, en ocasiones el valor estético y estilístico de los recursos -que son los que se busca aprovechar en mayor medida mediante la restauración $3 / 4$ puede significar la pérdida del valor y la significancia real de estos, pues quienes llevan a cabo estas restauraciones pueden caer en un ejercicio autónomo de la creatividad, de acuerdo con Sánchez (2013) y Checa (2012). 


\section{Caracterización general de la producción analizada}

Una vez diferenciadas las aportaciones y delimitadas en las líneas temáticas, se consideró pertinente efectuar un análisis referente a los trabajos. En primera instancia se valoró cuantitativamente su contribución a las líneas temáticas: $7.14 \%$ corresponde a la de bienes patrimoniales e identidad social; $32.14 \%$ a la de aprovechamiento y viabilidad de los bienes patrimoniales como recurso turístico; $28.58 \%$ a la de los imaginarios turísticos sobre los bienes patrimoniales, y $32.14 \%$ a la de recuperación y restauración de los bienes patrimoniales para su aprovechamiento turístico.

Se concluye que, para esta investigación, los trabajos enfocados al aprovechamiento y viabilidad de los bienes patrimoniales como recurso turístico, así como los de recuperación y restauración de los bienes patrimoniales, son los predominantes; por el contrario, aquellos centrados en los bienes patrimoniales e identidad social son los menos realizados.

En el aspecto metodológico concerniente a las investigaciones analizadas se observó que el enfoque cualitativo es el predominante, con $57.14 \%$, seguido de las metodologías mixtas con $25 \%$, y finalmente la perspectiva cuantitativa con $17.86 \%$.

Asimismo se identificaron las herramientas metodológicas utilizadas: para los estudios cualitativos, las más significativas fueron el análisis de contenido, la observación y la investigación documental; en algunos casos se emplearon otras como el focus group (grupo de enfoque), entrevistas a profundidad, inventarios, y matrices swot o DOFA (por sus siglas en inglés) y PEST. Para los estudios cuantitativos, las herramientas más usadas fueron el análisis de datos secundarios, encuestas e inventarios, apoyándose también de matrices swoT y PESTEL.

El siguiente elemento de la caracterización de la producción analizada es el lugar de procedencia y de estudio de las investigaciones (véase cuadro 1).

El cuadro 1 muestra el papel de los países en la elaboración de artículos científicos respecto al turismo cultural, en específico al aprovechamiento de los bienes culturales en el turismo. Los países donde la investigación del tema cobra mayor importancia son México, Malasia, Turquía y España, lo que puede significar que los modelos turísticos de la modalidad cultural tengan o puedan tener una fuerte influencia en ellos. Otra realidad que refleja esta situación es la relevancia de los bienes patrimoniales culturales locales, así como su conservación 
CUADRo 1. Número de investigaciones, lugares de procedencia y de estudio

\begin{tabular}{cl}
$\begin{array}{c}\text { Núm. de } \\
\text { investigaciones }\end{array}$ & \multicolumn{1}{c}{ Procedencia } \\
\hline 1 & Australia \\
4 & España \\
1 & Grecia \\
2 & Indonesia \\
5 & Malasia \\
6 & México \\
\hline 2 & República de Macedonia \\
1 & Suiza \\
\hline 1 & Tailandia \\
5 & Turquía \\
\hline
\end{tabular}

Fuente: Elaboración propia.

para su puesta en escena turística en esos países y regiones (Iberoamérica). El caso de Suiza, pese a ser el sitio donde la investigación tiene lugar, el estudio y todo lo relacionado con él concierne a España; por ello, la aportación recae en el área de estudio y no en el lugar de procedencia.

Por último, y como consecuencia del análisis anterior, se considera de interés una catalogación de las instituciones donde tienen lugar tales investigaciones (véase cuadro 2).

Es sabido que lo relacionado con el ámbito educativo y, por ende, las producciones científicas, así como las investigaciones sobre ciertos temas, varían de acuerdo con el contexto social de las poblaciones que las desarrollan. Esto no es tema del presente trabajo, sin embargo constituye uno de los factores determinantes para la investigación.

Los países que sobresalen son los mismos que tienen la mayor producción de investigaciones: Turquía, Malasia y España, pero en este caso lo que resalta es la pluralidad de instituciones en las que se realizan las investigaciones; en el caso de Malasia, a priori parece coincidir en la universidad, aunque en diferentes facultades. 
Cuadro 2. Instituciones y países de procedencia

\begin{tabular}{|c|c|}
\hline País & Instituciones \\
\hline Australia & Monash University \\
\hline España & $\begin{array}{l}\text { Universidad de Cádiz } \\
\text { Universidad de Barcelona } \\
\text { Universidad de Sevilla } \\
\text { Universidad de Valencia }\end{array}$ \\
\hline Grecia & Architect desa, stage designer dea Themistokleous \\
\hline Indonesia & $\begin{array}{l}\text { Urban and Regional Planning ITS } \\
\text { Urban and Regional Planning Department, Sepuluh Nopember } \\
\text { Institute of Technology }\end{array}$ \\
\hline Malasia & $\begin{array}{l}\text { Universiti Teknologi Malaysia } \\
\text { Faculty of Architecture, Planning and Surveying, Universiti } \\
\text { Teknologi MARA } \\
\text { Arshad Ayub Graduate School, Universiti Teknologi MARA } \\
\text { Taman Alam Melayu Nusantara (TAMAN) Faculty of } \\
\text { Architecture, Planning \& Surveying Universiti Tecknologi MARA }\end{array}$ \\
\hline México & $\begin{array}{l}\text { Universidad Veracruzana } \\
\text { Universidad Autónoma de Baja California } \\
\text { Universidad Autónoma Metropolitana } \\
\text { Universidad Autónoma del Estado de México }\end{array}$ \\
\hline $\begin{array}{l}\text { República } \\
\text { de } \\
\text { Macedonia }\end{array}$ & Faculty of Tourism and Hospitality, Ohrid University \\
\hline Suiza & Institute os Terrestrial Ecosystems \\
\hline Tailandia & Suan Sunandha Rajabhat University \\
\hline Turquía & $\begin{array}{l}\text { Faculty of Education, Ïnönü Univercity } \\
\text { Ege University, Faculty of Letters Department of Geography } \\
\text { Karabük University, Faculty of Arts, Geography Department } \\
\text { Ege University, Program of Tourism and Hotel Management } \\
\text { Campus of Yusuf Perin } \\
\text { Meram Anatolian Vocational Trade High School }\end{array}$ \\
\hline
\end{tabular}




\section{Conclusiones}

El turismo cultural es una de las modalidades más importantes para el turismo, además de una de las más antiguas. Por ello se dispone de investigaciones referentes al aprovechamiento de los bienes patrimoniales culturales en esta actividad.

Cada vez más se considera al turismo cultural, mediante el aprovechamiento de los bienes patrimoniales, como una alternativa factible de implementación en los destinos (maduros o no), ya que, según se ha demostrado en diversas investigaciones, ayuda a combatir o prevenir la estacionalidad turística de los destinos y atractivos, además de propiciar la diversificación no solo de los servicios enfocados a la actividad turística, sino también de los atractivos, ya que da pauta a crear, modernizar o adaptar nuevos modelos o atractivos para un mismo destino.

Por otra parte, al realizar la investigación documental, se observó que para integrar las subtipologías turísticas hay autores que consideran algunas modalidades (el turismo rural, por ejemplo) como una tipología similar al turismo cultural, y hay quienes las ven como una subcategoría de este. Por ello se han propuesto nuevos modelos en los cuales los bienes patrimoniales que se aprovechan en el turismo cultural no son de corte social, antropológico o arquitectónico, sino natural, como los paisajes naturales, los elementos de imagen urbana, e incluso la literatura, es decir, todo aquello que por su propia naturaleza o por la cercanía a otros recursos ya reconocidos pueda posicionarse en los imaginarios turísticos de los visitantes reales o potenciales.

Otro aspecto sobresaliente que resulta de esta investigación es el enfoque de identidad social con respecto a los bienes patrimoniales culturales antes de su aprovechamiento turístico. Así, se plantea que previo a su consideración en la escena turística, los elementos identificados deben ser valorados e interpretados dentro de un sistema de valores culturales de la comunidad en la que se encuentran, ya que del nivel de aceptación y de identificación por parte de la comunidad tanto de los recursos como del turismo, dependerá la posibilidad de éxito o fracaso como atractivos en su operación turística. Hoy en día solo se ponen a disposición del turismo aquellos bienes que presentan un valor histórico, social, cultural, arquitectónico o de cualquier otra índole, siempre y cuando se 
hayan desarrollado simbióticamente y demuestren o exalten la identidad cultural y colectiva de una comunidad.

La tendencia que revela este acercamiento al tema de los bienes patrimoniales es que las líneas temáticas más trabajadas son las vinculadas con el aprovechamiento turístico de los bienes patrimoniales, tanto en relación con su viabilidad como con su restauración, lo cual claramente corresponde a su naturaleza como segmento de mercado. A esta misma naturaleza concierne gran parte de los trabajos incluidos en la línea temática de los imaginarios, puesto que son varias las conexiones que hacen los autores entre imaginarios y posicionamiento. Por tanto, no es de extrañar que el tema de bienes patrimoniales e identidad social sea el menos desarrollado. Aunque se trata solo de una aproximación al estado de la investigación de los bienes patrimoniales, se considera que las líneas identificadas son válidas para caracterizar las tendencias relevantes sobre el tema.

El predomino de metodologías cualitativas constituye otro rasgo distintivo de esta temática, ya que la investigación turística es más cualitativa que cuantitativa. También resalta que este tema se muestra relevante no solo en los países europeos de tradición dominante sobre su legado patrimonial, sino además en países asiáticos que se encuentran investigando la puesta en valor de sus bienes patrimoniales (tanto culturales como naturales).

Aunque el caso de México puede estar sobreestimado por incluir una revista específica del país, se destaca que existe un interés creciente por el tema, visualizado por el trabajo de varias instituciones que se inclinan no solo por los bienes patrimoniales monumentales, sino también por los intangibles.

\section{Fuentes consultadas}

Akbulut, G. y Artvinli, E. (2011). Effects of Turikish raiway museum on cultural tourism. Procedia - Social and Behavioral Sciences, 19, 131-138.

André, M., Cortés, I. y López, J. (2003). Turismo cultural: cuando el recurso cultural supera al destino turístico. El caso de Figueres. En XII Simposio Internacional de Turismo y Ocio. Recuperado de https://www. bmi.gob.sv/pls/portal/docs/PAGE/BMI_HTMLS/BMI_PULSO_IMG/ TURISMO \% 20CULTURAL\%20FIGUERES\%20Y\%20DALI.PDF [2016, 2 de mayo]. 
Ataberk, E. y Baykal, F. (2011). Utilization of natural and cultural resources of Dikili (Izmir) for tourism. Procedia - Social and Behavioral Sciences, 19, 173-180.

Binti Abu Hassan, Z., Abdul Kadir bin Jailani, M. y Abd. Rahim, F. (2014). Assessing the situational analysis of heritage tourism industry in Melaka. Procedia - Social and Behavioral Sciences, 130, 28-36.

Checa, M. M. (2012). Aproximación turístico-cultural para un patrimonio en vías de extinción: la arquitectura histórica en madera de Chetumal, Quintana Roo. El Periplo Sustentable, 23, 49-78.

Chui Teo, C. B., Mohd Khan, N. R. y Abd Rahim, F. H. (2014). Understanding cultural heritage visitor behavior: The case of Melaka as world heritage city. Procedia - Social and Behavioral Sciences, 130, 1-10.

Conesa, H. M. (2010). The difficulties in the development of mining tourism projects: The case of La Unión District (SE Spain). PASOS. Revista de Turismo y Patrimonio Cultural, 4, 653-660.

Creative Nation: Commonwealth Cultural Policy (octubre, 1994). Department of Communications and the Arts, Australia. Recuperado de http://pandora.nla.gov.au/pan/21336/20031011-0000/www.nla.gov.au/creative. nation/contents.html [2016, 19 de abril].

Dwyer, L., Gill, A. y Seetaram, N. (2012). Handbook of Research Methods in Tourism Quantitative and Qualitative Approaches. En L. Dwyer et al., Handbook of Research Methods in Tourism Quantitative and Qualitative Approaches (pp. 443-445). Cheltenham: Edward Elgar.

Emekli, G. y Baykal, F. (2011). Opportunities of utilizing natural and cultural resources of Bornova (Izmir) through tourism. Procedia - Social and Behavioral Sciences, 19, 181-189.

Fernández Poncela, A. M. (2011). Conmemoraciones históricas, activación y posicionamiento turístico. Centenario, Bicentenario y Tricentenario en Chihuahua. El Periplo Sustentable, 21, 139-169.

García Jiménez, M. (2010). Patrimonio y herencia cultural: iescenarios de divergencia? Sphera Pública, número especial, 337-372.

Güngör, S. y Bozyiğit, R. (2011). Tourism potential of Gazipasa (Antalya - Turkey). Procedia - Social and Behavioral Sciences, 19, 231-239.

Hassan, H. (2014). The representation of Malaysian cultures in tourism brochures. Procedia - Social and Behavioral Sciences, 118, 140-151.

Hernández, R., Fernández, C. y Baptista, P. (2014). Metodología de la inves- 
tigación. En R. Hérnandez et al., Metodología de la investigación (pp. 422-451). Ciudad de México: McGraw-Hill.

Idajati, H. (2014). Cultural and tourism planning as tool for city revitalization: The case of study of Kalimas River, Surabaya-Indonesia. Procedia - Social and Behavioral Sciences, 135, 136-141.

Judd, D. R. (2003). El turismo urbano y la geografia de la ciudad. Eure, 29(87), 51-62.

Kaya, H. y Kurt, H. (2011). Contribution of concrete cultural constituents to the tourism potencial of Safranbolu. Procedia - Social and Behavioral Sciences, 19, 225-230.

Korunovski, S. y Marinoski, N. (2012). Cultural tourism in Ohrid as a selective form of tourism development. Procedia - Social and Behavioral Sciences, 44, 104-113.

Laws of Malaysia (2006). Act 645. National Heritage Act 2005. Kuala Lumpur: The Commissioner of Law Revision, Malaysia. Recuperado de http:// www.gtwhi.com.my/images/stories/files/NATIONAL \% 20HERITAGE\%20ACT\%202005.pdf [2016, 25 de mayo].

López, J. A., Arcila, M., Micossi, V. y Cano, M. (2013). Modelo-guía de valoración turística del patrimonio religioso: los templos de Jerez de la Frontera. PASOS. Revista de Turismo y Patrimonio Cultural, 11(1), 147-157.

Luekveerawattana, S. (2012). Cultural landscape for sustainable tourism. Case study of Amphawa community. Procedia - Social and Behavioral Sciences, 65, 387-396.

Mallor, E., González-Gallarza, M. y Fayos, T. (2013). ¿Qué es y cómo se mide el turismo cultural? Un estudio longitudinal con series temporales para el caso Español. PASOS. Revista de Turismo y Patrimonio Cultural, 11, 269-284.

Marinoski, N. y Korunovski, S. (2012). Tourism in Macedonia in changing environment. Procedia - Social and Behavioral Sciences, 44, 19-31.

Martha, L. y Kotzaki, A. (2014). Ancient greek drama and its architecture as a means to reinforce tourism in greece. Procedia - Social and Behavioral Sciences, 148, 573-578.

McIntosh, R. W., Goeldner, C. R. y Ritchie, J. R. B. (1995). Tourism: Principles, Practices, Philosophies (7 ${ }^{\text {a }}$ ed.). Nueva York: Jhon Wiley \& Sons. 
Mendoza, M. M., Umbral, M. E. y Arévalo, M. N. (2011). La interpretación del patrimonio, una herramienta para el profesional del turismo. El Periplo Sustentable, 20, 9-30.

Mestre, S. (2010). Reflexión sobre el impacto de los museos tarraconenses en el turismo. Alternativa al producto de sol y playa. PASOS. Revista de Turismo y Patrimonio Cultural, 8, 83-90.

Mohd, N. I., Ahmad, S. y Syed, S. M. H. (2013). Between tourism and intagible cultural heritage. Procedia - Social and Behavioral Sciences, 85, 411-420.

Orbasli, A. (2000). Tourists in Historic Towns: Urban Conservation and Heritage Management. Nueva York: E \& FN Spon Taylor and Francis Group.

Peñalver, M. T. (2002). La arquitectura industrial: patrimonio histórico y su utilización como recurso turístico. Cuadernos de Turismo, $10,155-166$.

Pillet, F. (2014). El paisaje literario y su relación con el turismo cultural. Cuadernos de Turismo, 33, 297-309.

Prats, L. (2003). Patrimonio + Turismo $=i$ Desarrollo? PASOS. Revista de Turismo y Patrimonio Cultural, 1(2), 127-136.

Prats, L. (2011). La viabilidad turística del patrimonio. PASOS. Revista de Turismo y Patrimonio Cultural, 9(2), 249-264.

Rahmawati, D., Supriharjo, R., Setiawan, R. P. y Pradinie, K. (2014). Community participation in heritage tourism for gresik resilience. Procedia - Social and Behavioral Sciences, 135, 142-146.

Ruiz, J. G., Martínez, O. C. y Verján, R. (2015). Valoración de atributos culturales e históricos en la imagen promocional de la ciudad de Tijuana como destino turístico. El Periplo Sustentable, 28, 31-58.

Sánchez, A. J. (2013). Patrimonio auténtico, turismo auténtico. Influencia de la cultura postmoderna en el concepto de restauración. PASOS. Revista de Turismo y Patrimonio Cultural, 11, 603-614.

Serrano, H. P., Zarza, M. P. y Serrano, C. (2013). Turismo cultural, transiciones en términos de género y su prospectiva. El Periplo Sustentable, 25, 135-158.

Smith, V. L. (1979). Hosts and Guests: The Anthropology of Tourism. Filadelfia: University of Pennsylvania Press.

Tovar, R. y Alvarado, C. (2010). Xochitepec. Una propuesta de desarrollo turístico cultural integral. El Periplo Sustentable, 19, 93-121. 
Tunbridge, J. E. y Ashworth, G. J. (1996). Dissonant Heritage. The Management of the Past as a Resource in Conflict. Chichester: John Wiley \& Sons.

Unesco. (1982). Conferencia Mundial sobre el Patrimonio Cultural. Definición. Patrimonio Cultural de la UNESCO, México, 1982. Recuperado de https://www.google.com.mx/l?sa $=t \& r c t=j \& q=\&$ esrc $=\mathrm{s} \&$ source $=$ web $\& c d=4 \& \mathrm{cad}=$ rja\&uact $=8 \& \mathrm{ved}=0$ ahUKEwjvyIbDu MnOAhUIKiYKHV3UBDgQFggsMAM\&url = http \%3A \%2F\%2Fmuseom aritimo.com \% 2Fadimra \% 2FActividades \% 2FPatrimonio \% 2520Cultural \%2FDefinicion \%2520de\%2520la \%2520UNESCO \%2520M\% [2015, 15 de noviembre].

Unesco. (2009). Lista del Patrimonio Mundial. Recuperado de http://portal. unesco.org/es/ev.php-URL_ID $=45692 \& U R L \_D O=$ DO_TOPIC\&URL_ SECTION = 201.html [2016, 28 de junio].

Unesco. (2013). Operational Guidelines for the Implementation of the World Heritage Convention. Recuperado de http://whc.unesco.org/archive/ opguide13-en.pdf [2015, 19 de octubre].

Universidad Interamericana para el Desarrollo (s. f.). Patrimonio Turístico. Recuperado de http://moodle2.unid.edu.mx/dts_cursos_mdl/lic/AET/ PT/S05/PT05_Lectura.pdf [2015, 10 de diciembre].

Velasco, M. (2009). Gestión turística del patrimonio cultural. Enfoques para un desarrollo sostenible del turismo cultural. Cuadernos de Turismo, 10, 237-253.

World Economic Forum (2015). The Travel \& Tourism Competitivness Report 2015. Recuperado de http://www3.weforum.org/docs/TT15/WEF_ Global_Travel\&Tourism_Report_2015.pdf [2015, 15 de octubre].

World Tourism Organization. (2008). Understanding Tourism: Basic Glossary. Recuperado de http://media.unwto.org/en/content/understandingtourism-basic-glossary. [2015, 2 de septiembre].

Zain, M. (2014). Translating the style of tourism promotional discourse: A cross cultural journey into stylescapes. Procedia - Social and Behavioral Sciences, 118, 503-510.

Zamil, A., Hafiz, I. y Abd Rashid, M. S. (2014). Identity of Malay Garden Desing to be promoted as the cultural tourism in Malaysia. Procedia - Social and Behavioral Sciences, 153, 298-307.

Zamudio Vega, L. S. (2012). La arquitectura en los imaginarios turísticos. RIPS. Revista de Investigaciones Políticas y Sociológicas, 11(2), 145-158. 